\title{
Avaliação do crescimento de espécies nativas plantadas em área de compensação florestal
} \author{
Charline Zangalli ${ }^{*}$ \\ ${ }^{1}$ Universidade do Estado de Santa Catarina, Avenida Luiz de Camões, 2090, Conta Dinheiro, Lages - SC, 88520-000 \\ 2 Universidade Federal de Santa Maria, Avenida Roraima, 1000, Camobi, Santa Maria - RS, 97105-900 \\ *Autor para correspondência: charlineeng @gmail.com \\ Recebido: Julho 2019/ Aceito: Novembro 2019/ Publicado: Dezembro 2019
}

Gabriel Loureço de Jesus ${ }^{1}$, Solon Jonas Longhi ${ }^{2}$, Klerysson Julio Farias ${ }^{1}$, Maria Raquel Kanieski ${ }^{1}$, Eliana Turmina ${ }^{1}$,

\section{Resumo}

As instalações de linhas de transmissão de energia elétrica geram impactos ambientais e estes podem ser minimizados por meio de medidas mitigadoras e compensatórias. Dentre as medidas de compensação florestal, destacam-se as técnicas utilizadas em programas de recuperação de áreas degradadas, como estratégia de reposição florestal. Neste contexto, este estudo teve como objetivo avaliar o crescimento de mudas de espécies florestais plantadas em uma área de compensação florestal, no Sul do Brasil. Foram amostradas 22 unidades amostrais de $100 \mathrm{~m}^{2}$, para a análise do incremento bimestral das mudas plantadas, sob duas formas de manejo (com e sem coroamento), durante um ano. Foram inventariados 105 indivíduos, distribuídos em 11 famílias e 21 espécies. Os dados de incremento em diâmetro do colo (DC), apresentaram normalidade (Shapiro-Wilk), homogeneidade (Bartlett) e confiabilidade (Acurácia seletiva). Estes apresentaram diferença significativa (Scott-Knott $(p<0,05))$. O agrupamento pelo dendrograma mostrou a formação de três agrupamentos pelo incremento em DC, confirmados pela análise discriminante. A correlação de Pearson apresentou correlação significativa, entre incremento e as variáveis meteorológicas avaliadas. Este estudo pode servir de subsídio para futuros plantios em área de recuperação, pois fornece conhecimento do padrão de crescimento das mudas e evidencia a necessidade da manutenção da área.

Palavras-chave: Linha de transmissão, Incremento diamétrico do colo, Correlação, Agrupamentos.

\section{Introdução}

As fontes de energia são extremamente importantes para o desenvolvimento e crescimento de um país. No Brasil, atualmente, há o uso de diversas fontes de energia, como energia hidroelétrica, eólica, petróleo, carvão mineral, biocombustíveis, gás natural e energia nuclear, sendo que $90 \%$ da energia utilizada no país provém de hidroelétricas (Pena 2018). Além das hidroelétricas, nos últimos anos, o setor de energia eólica apresentou um alto crescimento, tendo inúmero projetos contratados, visando um aumento de $450 \%$ em apenas cinco anos (Simas e Pacca 2013).

Diante disso, para a distribuição dessa energia da usina ao seu destino final se faz necessário o uso de linhas de transmissão. As instalações de linhas de transmissão geram impactos ambientais sobre o meio biótico, físico e socioeconômico e estes impactos são minimizados por meio de medidas mitigadoras e compensatórias (Tobouti e Santos 2014).

Como exemplo de medida de compensação ambiental tem-se a restauração de ecossistemas degradados. Esta tem foco não apenas em revegetação e sim de recuperar as áreas com ganhos de estabilidade das comunidades e ecossistemas. A restauração florestal promove o avanço temporal dos processos ecológicos, da estrutura e da dinâmica sucessional (Martins 2014). Para tanto, além da aplicação de técnicas de recuperação, se faz necessário o monitoramento e avaliação do crescimento das mudas (Brancalion et al. 2013). O crescimento das plantas é influenciado por diversas características, desde a interação com o ambiente (variáveis meteorológicas, edáficas, topográficas), além da competição com outras espécies (Kanieski et al. 2017).

Diversos trabalhos como Kanieski et al. (2017), Kanieski et al. (2013) e Zanon e Finger (2010), foram realizados com o intuito de avaliar a influência das variáveis meteorológicas no crescimento das espécies florestais. Porém, poucas informações se têm em relação ao crescimento das mudas em relação à forma de manejo, sendo que grande parte dos problemas associados às áreas de reposição florestal, se devem à falhas na manutenção e monitoramento. Embora o monitoramento e a avaliação sejam atividades fundamentais para o projeto de restauração, ainda existem algumas lacunas a serem superadas para que essas atividades possam ser incorporadas de forma rotineira no planejamento de ações de restauração, assim como, determinar indicadores ecológicos mais importantes para se estimar as chances de sucesso na restauração florestal (Brancalion et al. 2013).

A hipótese desse estudo é que mudas plantadas em área de compensação florestal na formação Floresta Ombrófila Mista apresentam correlação entre crescimento e variáveis meteorológicas de precipitação e temperatura, além do crescimento de mudas ser afetado pela forma de manejo, apresentando maior crescimento quando da presença de coroamento.

Em virtude do que foi mencionado o objetivo desse estudo foi avaliar o crescimento de espécies nativas plantadas em uma área em processo de recuperação referente à implantação da Linha de Transmissão 525 kV Salto Santiago - Itá - Nova Santa Rita, na formação de Floresta Ombrófila Mista no sul do Brasil.

\section{Material e Métodos}

$\mathrm{O}$ estudo foi realizado em área destinada à compensação florestal referente à LT $525 \mathrm{kV}$ Salto Santiago, Itá, Nova Santa Rita. A área pertence a Estação Ecológica (ESEC) Mata Preta, situada no município de Abelardo Luz - SC (Figura 1), nas coordenadas $26^{\circ} 28^{\prime} \mathrm{S}$ e $52^{\circ} 09^{\prime} \mathrm{W}$, a uma altitude de 776 m. O clima na região é classificado como $\mathrm{Cfb}$ (Alvares et al. 2013), com temperatura média anual em torno de $17,3{ }^{\circ} \mathrm{C}$ e precipitação média de $2.216 \mathrm{~mm} /$ ano (Climate - data. ORG 2017).

A área avaliada é de 4,28 ha, sendo que, antes de ser destinada à compensação florestal, esta área pertencia a domínio privado, com manejo de uso agrícola e pecuário. No fim de 2014 e início de 2015 iniciou-se na área as atividades de pré-implantação do projeto, realizando o cercamento da área, limpeza, combate a formigas e, por fim, o efetivo plantio das mudas nativas, sob responsabilidade de uma empresa de consultoria contratada pelo empreendimento para a realização do plantio. O plantio das mudas foi em linhas (3 x $2 \mathrm{~m})$ sem combinação dos grupos sucessionais, sendo que não há 
registro de como havia ficado à disposição das mudas no campo. Após o plantio a área não recebeu monitoramento ou manutenção.

Um ano após o plantio das mudas, foram alocadas de forma sistemática 22 unidades amostrais (UA) de $100 \mathrm{~m}^{2}$ (10 $\mathrm{x} 10 \mathrm{~m}$ ). Realizou-se avaliação bimestral de todas as mudas inventariadas por um período de um ano, período de avaliação que fornece informações cruciais de desenvolvimento inicial das mudas após um ano sem monitoramento e manutenção, em que foram coletadas as variáveis diâmetro do colo (DC) e altura (h). As UA foram divididas aleatoriamente em dois grupos: UA com mudas coroadas e UA não coroadas, caracterizando duas formas distintas de manejo.

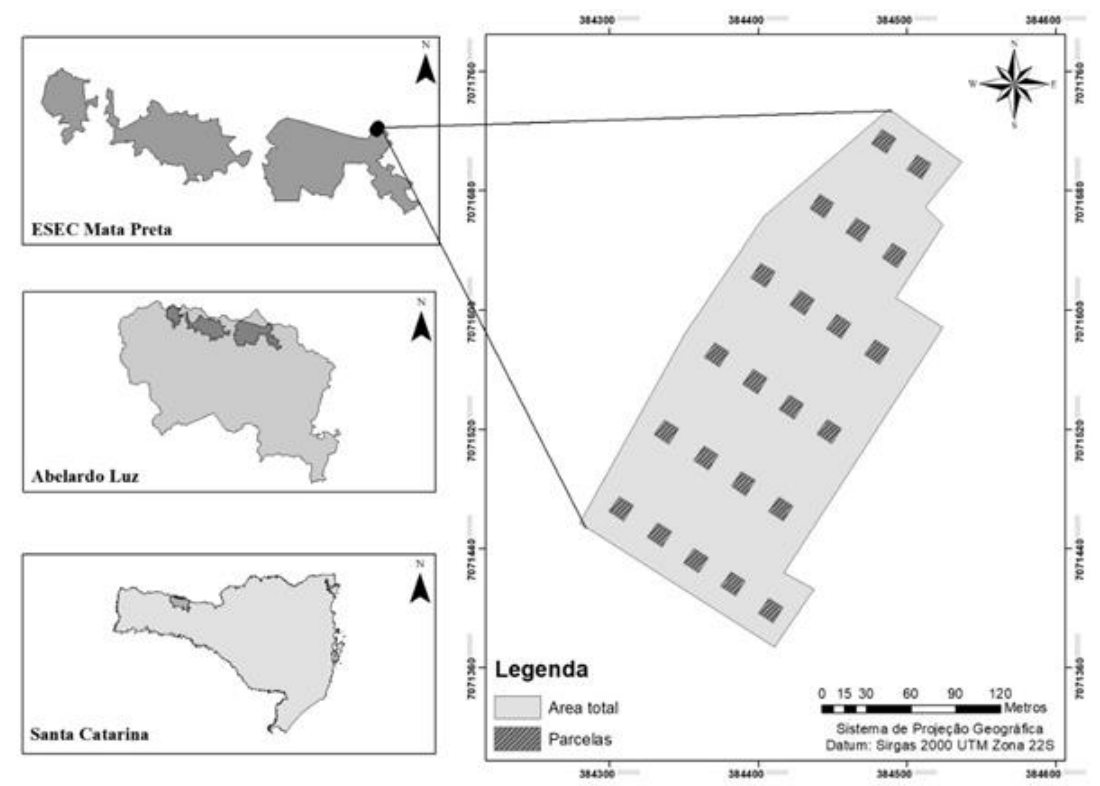

Figura 1. Localização da área de compensação florestal em Abelardo Luz, SC.

As espécies foram classificadas taxonomicamente de acordo com o sistema APG IV (Angiosperm Phylogeny Group 2016). A classificação do grupo ecológico foi realizada com base nos critérios propostos por Budowski (1970).

Para comparação visual entre o crescimento das mudas utilizou-se gráfico boxplot. Já com a finalidade de verificar as diferenças estatísticas no crescimento, foi realizado teste de normalidade de Shapiro-Wilk e teste de homogeneidade da variância de Bartlett, quando "F" foi significativo na ANOVA as médias de incremento corrente anual foram comparadas por meio do teste de Scott-Knott $(\mathrm{p}<0,05)$. A precisão do experimento foi medida por meio da acurácia seletiva (AS\%), valor que varia de 0 a 1 , sendo que quanto mais próximo de 1 mais confiável.

Para identificar correlações entre a variável de incremento em diâmetro do colo (DC) e as variáveis meteorológicas de precipitação e temperatura, foi realizada a avaliação da correlação de Pearson $(p<0,05)$. As análises foram realizadas por meio do aplicativo SISVAR (Ferreira 2011).

Os dados meteorológicos para correlação (Figura 2), foram obtidos junto ao CIRAM (Centro de Informações de Recursos Ambientais e de Hidrometeorologia de Santa Catarina), tendo como referência informações diárias de estação meteorológica da cidade de Chapecó, SC, estação mais próxima da área de estudo, localizada a aproximadamente $84 \mathrm{~km}$. Para verificar possíveis agrupamentos das espécies pelo Incremento Acumulado Anual, foi utilizada representação com Dendrograma, pelo método de Mahalanobis e distância de Ward para ligação. As análises foram realizadas utilizando a linguagem de programação R (R Development Core Team 2017), com o pacote vegan (Oksanen et al. 2016). Para testar a significância dos agrupamentos foi realizada uma Análise Discriminante, pelo método Discriminante Linear de Fisher, utilizando o software ( $)$ Copyright IBM Corporation, versão 20 (IBM SPSS 2011).

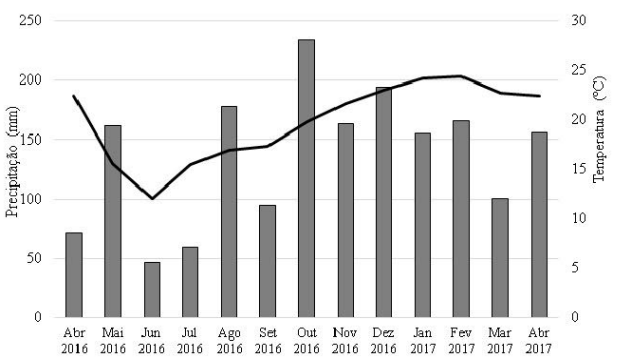

Figura 2. Dados meteorológicos de Precipitação acumulada (barras) e Temperatura média mensal (linhas), referentes ao mesmo período de avaliação das mudas plantadas.

\section{Resultados e Discussão}

Foram observados 105 indivíduos, distribuídos em 11 famílias, 18 gêneros e 21 espécies (Tabela 1). A família de maior riqueza foi Myrtaceae com sete espécies. Os gêneros de maior riqueza foram Handroanthus (2) e Psidium (2). Destaca-se o elevado número de indivíduos de Eugenia uniflora (14 indivíduos), Myrcianthes pungens (12 indivíduos) e Handroanthus chrysotrichus (12 indivíduos).

Em se tratando do grupo ecológico, os indivíduos estão distribuídos em quatro classes (Tabela 1), com destaque ao grupo das secundárias tardias (59\%) com mais de $50 \%$ dos indivíduos, seguido pelo grupo das secundárias iniciais (25\%) e pioneiras (15\%). Ferreira e Dias (2004), salientam que os modelos de recuperação, quando ressaltaram a indução da dinâmica de sucessão secundária, tem apresentado resultados muito favoráveis. 
Por se tratar de um plantio de mudas sem a combinação dos grupos sucessionais, o número de indivíduos inventariado de cada espécie variou bastante (Tabela 1), impossibilitando a análise estatística de algumas espécies com baixa representatividade. Bolfarine e Bussab (2005) complementam que, se a amostra não for representativa, as conclusões devem limitar-se à própria amostra, ou seja, apenas para este caso/local.

Tabela 1. Representação do número de indivíduos por espécie e classificação quando ao grupo ecológico.

\begin{tabular}{|c|c|c|c|c|}
\hline Família & Nome Científico & $\mathbf{C}$ & $\mathrm{NC}$ & GE \\
\hline Araucariaceae & Araucaria angustifolia (Bertol.) Kuntze & 2 & 3 & ST \\
\hline \multirow{3}{*}{ Bignoniaceae } & Jacaranda puberula Cham. & 1 & 1 & ST \\
\hline & Handroanthus chrysotrichus (Mart. ex A.DC.) Mattos & 7 & 5 & ST \\
\hline & Handroanthus heptaphyllus (Mart.) Mattos & 2 & 3 & ST \\
\hline Boraginaceae & Cordia americana (L.) Gottshling \& J.E.Mill. & 1 & 4 & PI \\
\hline Erythroxylaceae & Erythroxylum deciduum A.St.-Hil. & 1 & & SI \\
\hline Euphorbiaceae & Sapium glandulosum (L.) Morong & & 1 & PI \\
\hline \multirow{2}{*}{ Fabaceae } & Parapiptadenia rigida (Benth.) Brenan & 1 & 7 & PI \\
\hline & Inga vera Willd. & 1 & 2 & SI \\
\hline Lamiaceae & Vitex megapotamica (Spreng.) Moldenke & 2 & 3 & SI \\
\hline \multirow[t]{4}{*}{ Loganiaceae } & Strychnos brasiliensis (Spreng.) Mart. & 2 & 2 & SI \\
\hline & Psidium cattleianum Sabine & & 3 & SI \\
\hline & Eugenia involucrata DC. & 3 & 4 & ST \\
\hline & Psidium guajava Linnaeus, Carl von & 1 & & EX \\
\hline \multirow[t]{4}{*}{ Myrtaceae } & Myrcianthes pungens (O.Berg) D. Legrand & 4 & 8 & ST \\
\hline & Blepharocalyx salicifolius (Kunth) O.Berg & & 1 & $\mathrm{C}$ \\
\hline & Eugenia uniflora $\mathrm{L}$. & 8 & 6 & ST \\
\hline & Campomanesia guazumifolia (Cambess.) O.Berg. & 1 & 4 & ST \\
\hline \multirow[t]{2}{*}{ Rutaceae } & Zanthoxylum rhoifolium Lam. & & 1 & PI \\
\hline & Cupania vernalis Cambess. & 3 & 2 & SI \\
\hline Sapindaceae & Allophylus edulis (A.St.-Hil., Cambess. \& A. Juss.) Radlk. & 2 & 3 & SI \\
\hline Total & & 42 & 63 & \\
\hline
\end{tabular}

Legenda: $\mathrm{C}=$ Indivíduos coroados, $\mathrm{NC}=$ Indivíduos não coroados, $\mathrm{GE}=\mathrm{Grupo}$ ecológico, $\mathrm{PI}=$ Pioneira, SI= Secundária inicial, $\mathrm{ST}=$ Secundária tardia, $\mathrm{C}=$ Clímax.

Em relação ao crescimento das mudas, houve grande variação entre as espécies avaliadas e a condição de manutenção (com e sem coroamento). Esta variação se deve à adaptação, à fisiologia, à fitossanidade e ao grupo ecológico de cada espécie. A Figura 3 apresenta os valores de incremento acumulado anual, sendo possível visualizar a alta variação.

A maioria das espécies que receberam coroamento apresentam os maiores valores de incremento diamétrico, devido ao fato das plantas daninhas competirem por recursos com as mudas em. Este comportamento foi encontrado por Maciel et al. (2011) estudo do crescimento de mudas nativas, em que as mudas que receberam o manejo das plantas daninhas apresentam melhor desenvolvimento. Fragoso et al. (2016), encontraram baixos valores de crescimento diamétrico em estudo de crescimento de mudas nativas em área de restauração, resultado que foi justificado pela elevada quantidade de plantas daninhas presentes nas parcelas avaliadas.

Os dados de incremento bimestral apresentaram normalidade e homogeneidade. A precisão do experimento foi considerada alta ou confiável, sendo que a AS foi de 0,78. Com o teste de Scott - Knott (Tabela 2) foi possível verificar que as espécies $H$. chrysotrichus $\mathrm{C}$ e $\mathrm{NC}, H$. heptaphyllus $\mathrm{C}$, A. edulis $\mathrm{NC}$ e $V$. megapotamica $\mathrm{NC}$, apresentaram as maiores médias de incremento, não diferindo estatisticamente entre si e diferindo estatisticamente dos demais.

De acordo com Kanieski et al. (2012) o crescimento em diâmetro das espécies se dá de forma sazonal, tendo valores mais expressivos no verão até o início do outono e valores menos expressivos ou quase nulos nas épocas mais frias, o que está apresentado nos valores de incremento bimestral na Tabela 2 e de correlação com variáveis meteorológicas na Tabela 3. Figueiredo-Filho et al. (2008); Fragoso et al. (2016); Kanieski et al. (2017) ao avaliarem o incremento diamétrico de espécies também evidenciaram este comportamento.

Na Tabela 3 podem ser observadas as Correlações de Pearson entre o crescimento das espécies e as variáveis meteorológicas de precipitação acumulada e temperatura média, sendo que todos os valores foram significativos a $\mathrm{p}<0,05$. Algumas espécies apresentaram valores próximos a zero (0), o que demonstra que algumas espécies são mais sensíveis a outras variáveis, não apresentadas nesse estudo, como o comportamento fenológico e as variáveis bioclimáticas. 


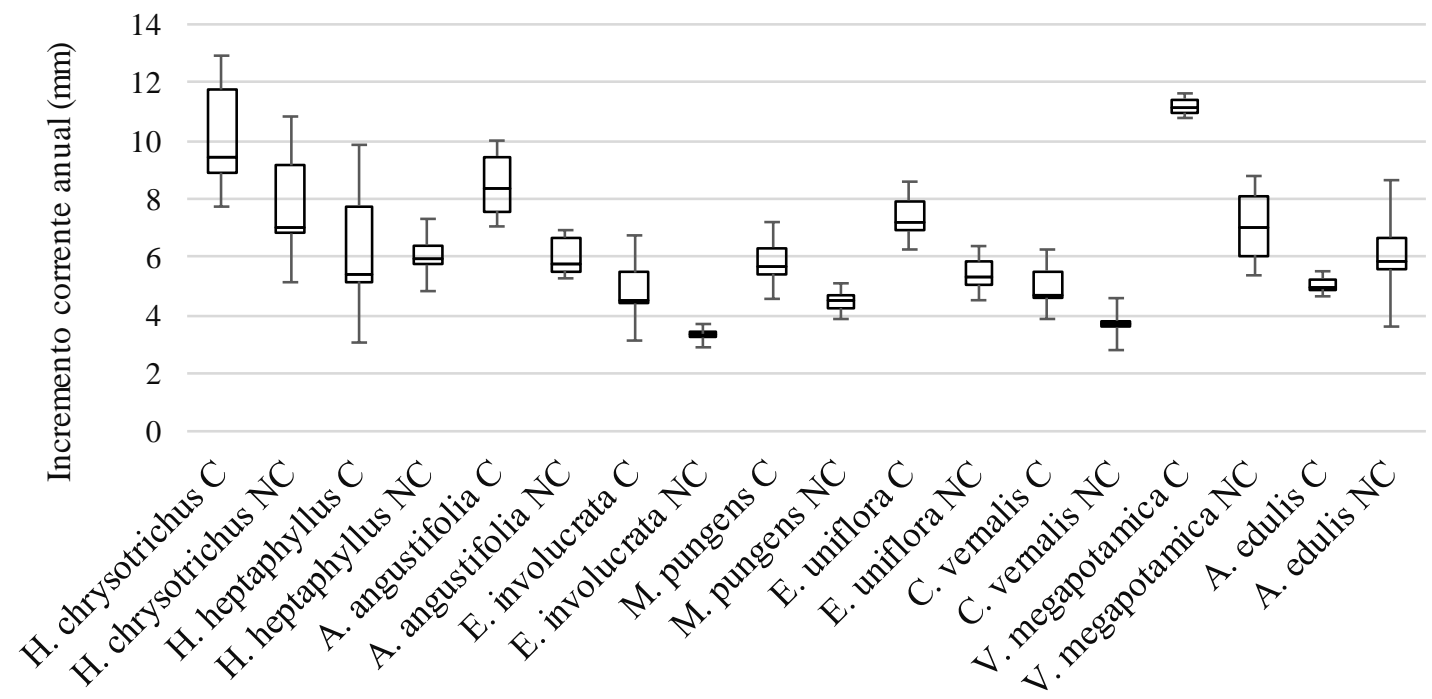

Figura 3. Gráfico Boxplot para as espécies com representatividade estatística, avaliadas por categoria (Coroada e Não coroada), em ordem decrescente do incremento corrente anual.

Tabela 2. Representação do resultado do teste de médias (Scott - Knott) para a variável de incremento corrente anual, para as espécies com representatividade nas duas categorias.

\begin{tabular}{|c|c|c|c|c|c|c|c|c|}
\hline Espécie & $04 / 16$ & $06 / 16$ & $08 / 16$ & $10 / 16$ & $12 / 16$ & $02 / 17$ & $04 / 17$ & $\begin{array}{l}\text { Incremento } \\
\text { acumulado } \\
\text { anual (mm) }\end{array}$ \\
\hline H. heptaphyllus $\mathrm{C}$ & 0 & 0,83 & 0,15 & 0,14 & 1,15 & 2,49 & 0,86 & $5,60 \mathrm{a}^{*}$ \\
\hline H. heptaphyllus NC & 0 & 0,18 & 0,18 & 0,11 & 0,12 & 0,69 & 0,56 & $1,84 \mathrm{~b}$ \\
\hline H. chrysotrichus $\mathrm{C}$ & 0 & 1,07 & 0,58 & 0,23 & 1,88 & 0,94 & 0,70 & $5,38 \mathrm{a}$ \\
\hline H.chrysotrichus NC & 0 & 0,78 & 0,30 & 0,09 & 1,07 & 2,05 & 0,66 & $4,95 \mathrm{a}$ \\
\hline A. edulis $\mathrm{C}$ & 0 & 0,13 & 0,01 & 0,04 & 0,10 & 0,44 & 0,03 & $0,75 \mathrm{~b}$ \\
\hline A. edulis NC & 0 & 0,69 & 0,35 & 0,07 & 0,41 & 0,85 & 1,55 & $3,93 \mathrm{a}$ \\
\hline V. megapotamica $\mathrm{C}$ & 0 & 0,60 & 0,25 & 0,05 & 0,20 & 0,22 & 0,08 & $1,38 \mathrm{~b}$ \\
\hline V.megapotamica $\mathrm{NC}$ & 0 & 0,03 & 1,39 & 0,29 & 0,84 & 0,39 & 0,51 & $3,44 \mathrm{a}$ \\
\hline A. angustifolia $\mathrm{C}$ & 0 & 0,10 & 0,30 & 0,64 & 0,63 & 0,95 & 0,05 & $2,67 \mathrm{~b}$ \\
\hline A. angustifolia $\mathrm{NC}$ & 0 & 0,64 & 0,06 & 0,18 & 0,80 & 0,25 & 0,14 & $2,06 \mathrm{~b}$ \\
\hline E. involucrata $\mathrm{C}$ & 0 & 0,04 & 0,05 & 0,03 & 0,49 & 1,03 & 0,77 & $2,40 \mathrm{~b}$ \\
\hline E. involucrata $\mathrm{NC}$ & 0 & 0,09 & 0,13 & 0,01 & 0,01 & 0,08 & 0,30 & $0,62 \mathrm{~b}$ \\
\hline M. pungens $\mathrm{C}$ & 0 & 0,21 & 0,26 & 0,09 & 0,49 & 0,41 & 0,66 & $2,11 \mathrm{~b}$ \\
\hline M. pungens $\mathrm{NC}$ & 0 & 0,17 & 0,38 & 0,09 & 0,09 & 0,19 & 0,28 & $1,20 \mathrm{~b}$ \\
\hline E. uniflora $\mathrm{C}$ & 0 & 0,23 & 0,37 & 0,12 & 0,60 & 0,29 & 0,50 & $2,10 \mathrm{~b}$ \\
\hline E. uniflora $\mathrm{NC}$ & 0 & 0,16 & 0,24 & 0,12 & 0,43 & 0,26 & 0,40 & $1,61 \mathrm{~b}$ \\
\hline C. vernalis $\mathrm{C}$ & 0 & 0,11 & 0,06 & 0,04 & 0,75 & 0,22 & 0,62 & $1,78 \mathrm{~b}$ \\
\hline C. vernalis $\mathrm{NC}$ & 0 & 0,05 & 0,08 & 0,12 & 0,04 & 0,07 & 0,75 & $1,09 \mathrm{~b}$ \\
\hline
\end{tabular}

* Letras diferentes representam diferença significativa pelo teste de Scott e Knott $(p<0,05)$.

O fato da espécie $A$. angustifolia ter apresentado valor de correlação negativo ou muito baixo com a variável de precipitação, está relacionado à ecologia da espécie, devido não ser adaptada em áreas de brejos ou com saturação de umidade no solo. Segundo Blum (1977) em estudo da ecologia da $A$. angustifolia, demonstrou que esta não tolera solos como água estagnada ou lençol freático alto. Outros autores afirmam que a araucaria necessita de solos bem drenados para apresentar um bom crescimento (Silva et al. 2001; Gerhardt et al. 2001; Carvalho 2008).
A precipitação é um dos fatores mais limitante ao desenvolvimento de espécies florestais, pois a água controla diversas funções fisiológicas desde a absorção de nutrientes as reações da fotossíntese (Landsberg 2003). A temperatura também possui impacto considerável no crescimento das plantas por ser um fator definitivo da velocidade da taxa em que ocorrem os processos metabólicos, influenciando diretamente na fotossíntese e na respiração celular (Landsberg e Sands 2011), sendo ambos, precipitação e temperatura, essenciais para o desenvolvimento das espécies florestais. 
Tabela 3. Correlação entre o incremento médio bimestral e as variáveis meteorológicas precipitação acumulada (mm) e temperatura média $\left({ }^{\circ} \mathrm{C}\right)$, nas duas categorias (coroadas e não coroadas).

\begin{tabular}{|c|c|c|}
\hline \multirow{2}{*}{ Espécies } & \multicolumn{2}{|c|}{ Variáveis Meteorológicas } \\
\hline & Precipitação & Temperatura \\
\hline Allophylus edulis $\mathrm{C}$ & $0,286^{*}$ & $0,468^{*}$ \\
\hline Allophylus edulis NC & $0,908^{*}$ & $0,408^{*}$ \\
\hline Araucaria angustifolia $\mathrm{C}$ & $-0,284 *$ & $0,551^{*}$ \\
\hline Araucaria angustifolia $\mathrm{NC}$ & $0,064 *$ & $-0,062 *$ \\
\hline Cupania vernalis $\mathrm{C}$ & $0,604 *$ & $0,632 *$ \\
\hline Cupania vernalis $\mathrm{NC}$ & $0,584 *$ & $0,346^{*}$ \\
\hline Eugenia involucrata $\mathrm{C}$ & $0,730 *$ & $0,896^{*}$ \\
\hline Eugenia involucrata $\mathrm{NC}$ & $0,709^{*}$ & $0,123^{*}$ \\
\hline Eugenia uniflora $\mathrm{C}$ & $0,636^{*}$ & $0,477 *$ \\
\hline Eugenia uniflora $\mathrm{NC}$ & $0,704 *$ & $0,645^{*}$ \\
\hline Handroanthus chrysotrichus $\mathrm{C}$ & $0,385^{*}$ & $0,263^{*}$ \\
\hline Handroanthus chrysotrichus NC & $0,546^{*}$ & $0,611^{*}$ \\
\hline Handroanthus heptaphyllus $\mathrm{C}$ & $0,554 *$ & $0,659^{*}$ \\
\hline Handroanthus heptaphyllus NC & $0,711^{*}$ & $0,661^{*}$ \\
\hline Myrcianthes pungens $\mathrm{C}$ & $0,885^{*}$ & $0,704^{*}$ \\
\hline Myrcianthes pungens $\mathrm{NC}$ & $0,386^{*}$ & $-0,162 *$ \\
\hline Vitex megapotamica $\mathrm{C}$ & $0,083 *$ & $-0,655^{*}$ \\
\hline Vitex megapotamica $\mathrm{NC}$ & $0,021^{*}$ & $0,035^{*}$ \\
\hline
\end{tabular}

*Correlação de Pearson significativa a um nível de $0,05(p<0,05)$.

A Figura 4 apresenta o dendrograma de agrupamento pelo Incremento Acumulado Anual. Houve diferenciação com a formação de três grandes grupos, o primeiro formado pelas espécies que obtiveram os maiores valores de incremento, o segundo, formado pelas espécies que obtiveram incremento médio e o terceiro com as espécies que obtiveram o menor crescimento entre as espécies avaliadas.

$\mathrm{O}$ primeiro agrupamento tem como funções principais recobrir o solo minimizando o efeito de erosão (Pereira e Rodrigues 2012; Silva et al. 2016), deposição de detritos vegetais, sendo responsável por restituir a fertilidade do solo e a ciclagem de nutrientes (Kageyama e Gandara, 2005), além de propiciar a menor incidência de luz direta no solo. Deste agrupamento a espécie que mais se destacou foi Inga vera, por apresentar o maior valor de incremento sob as duas condições de manejo, sendo a espécie que teve o melhor desenvolvimento na área. Além disso, esta é fixadora de nitrogênio, considerada ideal para a recuperação de áreas, aumentando a oferta de alimento para a avifauna.

O segundo agrupamento possuiu a maior parte das espécies classificadas como secundárias tardias e iniciais, com destaque para $H$. heptaphyllus e $J$. puberula, com os maiores incrementos. Segundo Grings e Brack (2011) a espécie de $H$. heptaphyllus é recomendada em plantios florestais e em áreas de recuperação. A espécie $J$. puberula também foi recomenda por Almeida et al. (2005); Ferreira et al. (2013); como uma espécie potencial para plantio em áreas de recuperação.

O terceiro grupo e maior grupo, apresentou espécies de todos os grupos sucessionais e abrangeu a única espécie clímax B. salicifolius. Kanieski et al. (2013) concluiu que esta é uma espécie potencial a ser utilizada em área degradada, apresentando bom desenvolvimento. Além destas espécies destaca-se também neste grupo de crescimento rápido, a presença de espécies classificadas como pioneiras por Budowski (1970); Silva et al. (2003); Backes e Irgang (2004).
Já autores como Longhi (1995); Vaccaro et al. (1999); Carvalho (2008); Aquino e Barbosa (2009); consideram as espécies $P$. rigida, $C$. americana e $Z$. rhoifolium, como espécies de crescimento lento a moderado, sendo comuns em clareiras e áreas abertas, sendo classificadas como secundárias inicias ou até mesmo tardias. Fato que ficou evidente no presente estudo por meio do dendrograma, em que essas espécies puderam ser consideradas com crescimento intermediário ou moderado, com grande potencial de colonizar áreas, sendo indicadas em projetos de recuperação.

A Análise de Discriminante, realizada para comprovar os três grupos formados, pelo teste de igualdade de médias do incremento corrente anual, demonstrou que existem diferenças entre os grupos, pois o Teste Lambda de Wilks, que varia de 0 a 1 , foi baixo $(0,237)$. Valor encontrado para o teste $\mathrm{M}$ de Box foi de 0,143 foi maior que o nível de significância $\alpha$, que é de 0,05 o que indica que as matrizes de covariâncias dos três grupos são homogêneas, requisito estatístico para a aplicação desta análise.

Foi obtida uma única função discriminante para classificar os tratamentos em função da variável avaliada (incremento corrente anual), para representar $100 \%$ da variância. O alto valor $(0,873)$ da correlação canônica da função indica alto grau de associação entre esta função e os grupos de espécies (Tabela 4).

O teste Lambda de Wilks (Tabela 4) testa a significância das funções discriminantes o que demonstrou que a função discriminante é altamente significativa $(p<0,001)$ (Nóbrega 2010). A percentagem de classificação correta das avaliações em seus respectivos grupos foi de $95,2 \%$, indicando que existe diferenças entre os três grupos de espécies (Tabela 5). Apenas uma espécie ( $V$. megapotamica) do Grupo 2 (crescimento médio), deveria pertencer ao Grupo 3, que possui menores incrementos. 
Pioneira

Secundária inicial

Secundária tardia

Climax

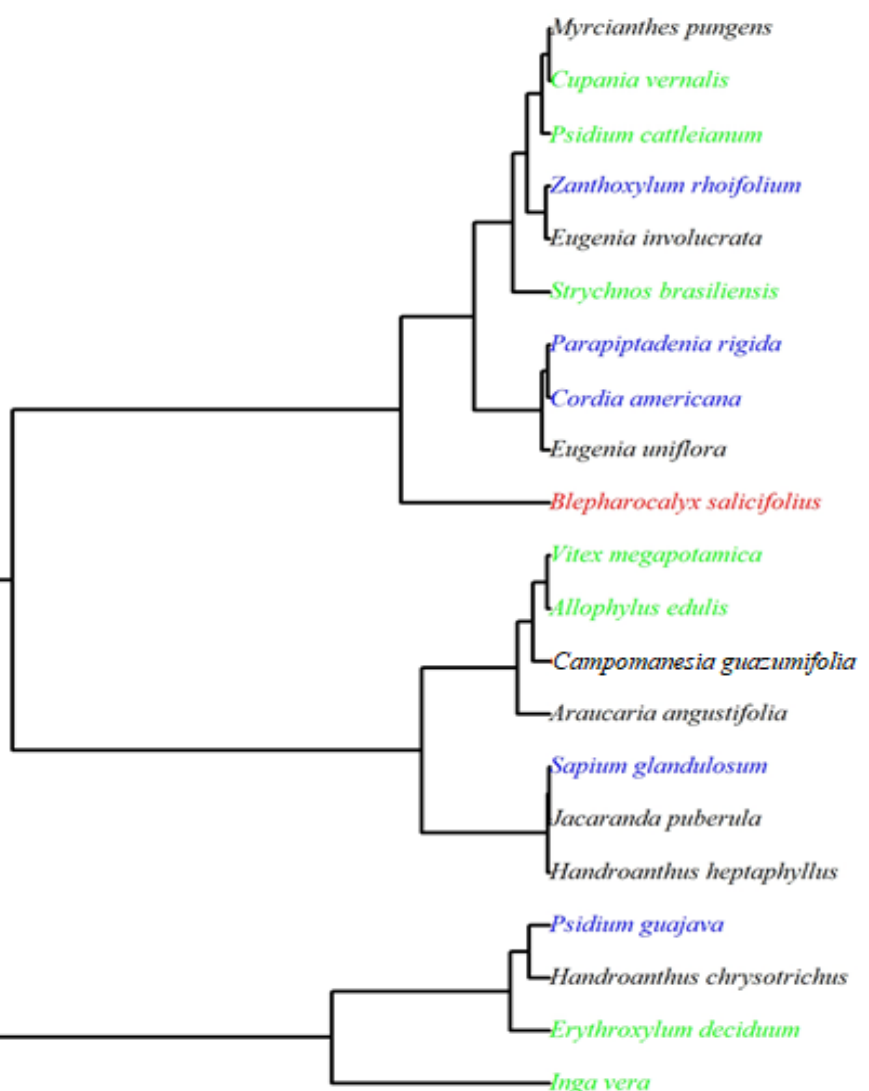

Figura 4. Dendrograma de agrupamento das espécies plantadas pelo ICA (Incremento Acumulado Anual), em área de recuperação florestal na localidade de Abelardo Luz, SC.

Tabela 4 - Estatística da seleção das funções discriminantes canônicas.

\begin{tabular}{|c|c|c|c|c|}
\hline \multicolumn{5}{|l|}{ Autovalores } \\
\hline Função & Autovalor & \% de variação & $\%$ cumulativa & $\begin{array}{l}\text { Correlação } \\
\text { canônica }\end{array}$ \\
\hline 1 & 3,215 & 100,0 & 100,0 & 0,873 \\
\hline \multicolumn{5}{|l|}{ Lambda de Wilks } \\
\hline Teste de funções & Lambda de Wilks & Chi-quadrado & $\mathbf{d f}^{*} *$ & Sig. \\
\hline 1 & 0,237 & 25,8952 & 2 & 0,000 \\
\hline
\end{tabular}

*df: Grau de liberdade.

Tabela 5. Classificação das mudas avaliadas em seus respectivos grupos de espécies.

\begin{tabular}{|c|c|c|c|c|c|c|}
\hline \multicolumn{7}{|c|}{ Associação ao Grupo } \\
\hline & & Grupos & 1 & 2 & 3 & Total \\
\hline \multirow{6}{*}{ Original } & \multirow{3}{*}{ Contagem } & 1 & 4 & 0 & 0 & 4 \\
\hline & & 2 & 0 & 6 & 1 & 7 \\
\hline & & 3 & 0 & 0 & 10 & 10 \\
\hline & \multirow{3}{*}{$\%$} & 1 & 100,0 & 0,0 & 0,0 & 100,0 \\
\hline & & 2 & 0,0 & 85,7 & 14,3 & 100,0 \\
\hline & & 3 & 0,0 & 0,0 & 100,0 & 100,0 \\
\hline
\end{tabular}

Observação: 95,2\% de casos originais foram corretamente classificados.

\section{Conclusões}

- Mesmo um ano após a implantação do projeto de reposição florestal como medida de compensação, sem manutenção, as espécies estudadas apresentaram elevado crescimento, diferindo de acordo com a espécie e a forma de manejo;

- As mudas apresentaram incremento diamétrico distinto quando da presença e ausência de coroamento, em que, as mudas com coroamento apresentaram maiores incrementos, demonstrando a eficiência da atividade e na necessidade de continuação de um monitoramento na área;
- O crescimento das mudas é influenciado pelas condições meteorológicas de precipitação e temperatura sendo que todas as espécies apresentaram correlação significativa;

- Este estudo serve de subsídio para futuros plantios em área de recuperação, pois fornece conhecimento do padrão de crescimento das mudas em relação aos dados meteorológicos e a necessidade de manutenção das mudas nas fases iniciais de crescimento, destacando-se também a necessidade e a importância de novos estudos relacionados. 
Referências

Almeida LS, Maia N, Ortega AR, Angelo AC (2005) Crescimento de mudas de Jacaranda puberula Cham. em viveiro submetidas a diferentes níveis de luminosidade. Ciência Florestal, 15 (3): 322-329. doi: $10.5902 / 198050981870$

Alvares CA, Stape JL, Sentelhas PC, Moraes G, Lonardo J, Sparoveck G (2013) Köppen's climate classification map for Brazil. Meteorologische Zeitschrift, 22 (6): 711-728. doi: 10.1127/0941-2948/2013/0507

Angiosperm Phylogeny Group (2016) An update of the Angiosperm Phy logeny Group classification for the orders and families of flowering plants: APG IV. Botanical Journal of the Linnean Society, 181 (2). doi: 10.1111/boj. 12385

Aquino C, Barbosa LM (2009) Classes sucessionais e síndromes de dispersão de espécies arbóreas e arbustivas existentes em vegetação ciliar remanescente (Conchal, SP), como subsídio para avaliar o potencial do fragmento como fonte de propágulos para enriquecimento de áreas revegetadas no Rio Mogi-Guaçu, SP. Revista Árvore, 33 (2): 349-358. doi: 10.1590/S0100-67622009000200016

Backes P, Irgang B (2004) Mata Atlântica: as árvores e a paisagem. Porto Alegre: Paisagem do Sul.

Blum WEH (1977) Ecologia da Araucaria angustifolia e futuras condições de reflorestamento no Sul do Brasil. Brasil Madeira, 7 (1): 10-12.

Brancalion PHS, Viani RAG, Rodrigues RR, Gandolfi S (2013) Avaliação e Monitoramento de Áreas em Processo de Restauração. In: Martins SV (ed) Restauração ecológica de ecossistemas degradados. Viçosa: Universidade Federal de Viçosa - UFV.

Bolfarine H, Bussab WO (2005) Elementos de amostragem. São Paulo: Edgar Blüchar, 274 p.

Budowski G (1970) The distinction between old secondary and climax species in tropical central american lowland forests. Journal of Tropical Ecology, 11(1): 44-48. doi: $10.1017 /$ S0266467400003989

Carvalho PER (2008) Espécies arbóreas brasileiras. Coleção Espécies Arbóreas Brasileiras, 3 ed. Colombo: Embrapa Florestas.

Climate - Data. ORG. Clima: Abelardo Luz - SC (2017) Disponível em: <https://pt.climatedata.org/location/43640/>. Acesso em: 02 Jan. 2018.

Ferreira DAC, Dias HCT (2004) Situação atual da mata ciliar do Ribeirão São Bartolomeu em Viçosa, MG. Revista Árvore, 28 (4): 617-623. doi: 10.1590/S010067622004000400016

Ferreira DF (2011) Sisvar: a computer statistical analysis system. Ciência e Agrotecnologia (UFLA), 35 (6): 1039 1042. doi: 10.1590/S1413-70542011000600001

Ferreira PI, Gomes JP, BatistaF, Bernardi AP, Costa NCFda, Bortoluzzi RLdaC, Mantovani A (2013) Espécies Potenciais para Recuperação de Áreas de Preservação Permanente no Planalto Catarinense. Floresta e Ambiente, 20 (2): 173-182. doi: 10.4322/floram.2013.003
Figueiredo-Filho A, Rode R, Figueiredo DJ de, Amaral SM (2008) Seasonal diameter increment for 7 species from an Ombrophyllous Mixed Forest, Southern state of Paraná, Brazil. Floresta, 38 (3): 527-543. doi: 10.5380/rf.v38i3.12424

Fragoso ROde, Temponi LG, Pereira DC, Guimarães ATB (2016) Recuperação de área degradada no domínio floresta estacional semidecidual sob diferentes tratamentos. Ciência Florestal, 26 (3): 699-711. doi: 10.5902/1980509824194

Gerhardt EJ, Finger CAG, Longhi SJ, Schumacher MV (2001) Contribuição da análise multivariada na classificação de sítios em povoamentos de Araucaria angustifolia (Bert.) O. Ktze., baseada nos fatores físicos e morfológicos do solo e no conteúdo de nutrientes da serapilheira. Ciência Florestal, v. 11, n. 2, p. 41-57. doi: 10.5902/19805098

Grings M, Brack P (2011) Handroanthus heptaphyllus - IpêRoxo. In: Coradin L, Siminski A, Reis A (ed) Espécies nativas da flora brasileira de valor econômico atual e potencial: plantas para o futuro, região Sul. Brasília, DF: Ministério do Meio Ambiente.

IBM SPSS (2011) C) Copyright IBM Corporation, versão 20. USA.

Kagey ama PY, Gandara FB (2005) Resultados do programa de restauração com espécies arbóreas nativas do convênio Esalq/USP e CESP. In: Galvão APM, Porfírio-da-Silva V (ed) Restauração florestal: fundamentos e estudos de caso. Colombo: EMBRAPA.

Kanieski MR, Galvão F, Santos TL, Milani JEF, Botosso PC (2017) Parâmetros Climáticos e Incremento Diamétrico de Espécies Florestais em Floresta Aluvial no Sul do Brasil. Floresta e Ambiente, 24 (1): 1-11. doi: 10.1590/21798087.124814

Kanieski MR, Longhi-Santos T, Milani JEdeF, Miranda BP, Galvão F, Botosso PC, Roderjan CV (2013) Crescimento diamétrico de Blepharocalyx salicifolius em remanescente de floresta ombrófila mista aluvial, Paraná. Floresta e Ambiente, 20(2): 197-206. doi: 10.4322/floram.2013.007

Kanieski MR, Santos TL, Graf-Neto J, Souza T, Galvão F, Roderjan CV (2012) Influência da Precipitação e da Temperatura no Incremento Diamétrico de Espécies Floreastais Aluviais em Araucária-PR. Floresta e Ambiente, 19 (1): 17-25. doi: 10.4322/floram.2012.003

Landsberg J, Sands P (2011) Phy siological ecology of forest production: principles, processes, and models. Amsterdam: Elsevier. Academic.

Landsberg J (2003) Phy siology in forest models: history and the future. London: FBMIS

Longhi RA (1995) Livro das árvores: árvores e arvoretas do Sul. Porto Alegre: L PM.

Maciel CDG, Poletine JP, Alves IM, Raimondi MA, Rodrigues M, Bueno RR, Costa RSda (2011) Coroamento no controle de plantas daninhas e desenvolvimento inicial de espécies florestais nativas. Semina: Ciências Agrárias, 32 (1): 119-128. doi: 10.5433/1679-0359.2011v32n1p 119 
Martins SV (2014) Recuperação de matas ciliares. 3. ed. Viçosa, MG: Aprenda Fácil.

Nóbrega DM (2010) Análise discriminante utilizando o software SPSS. Monografia (Conclusão de Curso de Bacharelado em estatística), Universidade Estadual da Paraíba, Campina Grande, 53p.

Oksanen J, Blanchet FG, Kindt R (2016) Vegan: community ecology package. R package version, (1): 8-8.

Pena RFA (2018) Fontes de Energia do Brasil. Disponível em: $<$ http://mundoeducacao.bol.uol.com.br/geografia/fonte s-energia-brasil.htm>. Acesso em: 21 Fev. 2018.

Pereira JS, Rodrigues SC (2012) Crescimento de espécies arbóreas utilizadas na recuperação de área degradada. Revista Caminhos de Geografia, 13 (41): 102-110.

R Development Core Team R: A language and environment for statistical computing. R Foundation for Statistical Computing. Disponível em: <http://www.Rproject.org Acesso em: 05 mai. 2017.

Silva AF, Oliveira RV, Santos NRL, Paula A (2003) Composição florística e grupos ecológicos das espécies de um trecho de floresta semidecídua submontana da fazenda São Geraldo, Viçosa-MG. Revista Árvore, 27 (6): 311-319. doi: 10.1590/S0100-67622003000300006

Silva H Dda, Bellote AFJ, Ferreira CA, Bognola IA (2001) Recomendação de solos para Araucaria angustifolia com base nas suas propriedades físicas e químicas. Boletim de Pesquisa Florestal, 1 (43): 61-74.

Silva KAde, Martins SV, Miranda Neto A, Demolinari RdeA, Lopes AT (2016) Restauração Florestal de uma mina de bauxita: avaliação do desenvolvimento das espécies arbóreas plantadas. Floresta e Ambiente, 23 (3): 309-319. doi: 10.1590/2179-8087.142515

Simas M, Pacca S (2013) Energia eólica, geração de empregos e desenvolvimento sustentável. Estudos Avançados, 27 (77). doi: 10.1590/S010340142013000100008

Tobouti AK, Santos VLP (2014) Impactos ambientais causados na implantação de linhas de transmissão no Brasil. Caderno Meio Ambiente e Sustentabilidade, 4 (3).

Vaccaro S, Longhi SJ, Brena DA (1999) Aspectos da composição florística e categorias sucessionais do estrato arbóreo de três subseres de uma floresta estacional decidual, no Município de Santa Tereza - RS. Ciência Florestal, 9 (1): 1-18. doi: 10.5902/19805098360

Zanon MLN, Finger CAG (2010) Relação de variáveis meteorológicas com o crescimento das árvores de Araucaria angustifolia (Bertol.) Kuntze em povoamentos implantados. Ciência Florestal, 20 (3): 467-476. doi: $10.5902 / 198050982061$ 